\title{
GROUPS OF MATRICES WITH INTEGER EIGENVALUES
}

\author{
M. R. FREISLICH
}

(Received 26 June 1969)

Communicated by G. E. Wall

\section{1}

Let $F$ be an algebraic number field, and $S$ a subgroup of the general linear group $G L(n, F)$. We shall call $S$ a $U$-group if $S$ satisfies the condition $(U)$ : Every $x \in S$ is a matrix all of whose eigenvalues are algebraic integers. This is equivalent to either of the following conditions:

a) the eigenvalues of each matrix $x$ are all units as algebraic numbers;

b) the characteristic polynomial for $x$ has all its coefficients integers in $F$.

In particular, then, every group of matrices with entries in the integers of $F$ is a $U$-group.

Our aim is to examine the structure of completely reducible soluble $U$-groups. We use the results given by Suprunenko [1] for soluble and nilpotent linear groups, and obtain some special conditions that must be satisfied by completely reducible soluble $U$-groups. We show that such groups are polycyclic, and we obtain some arithmetical conditions that must be satisfied by primitive irreducible soluble $U$-groups, depending on the degree of the group and the field $F$. The results obtained depend on results for irreducible abelian and nilpotent $U$-groups, which we examine separately.

\section{Abelian $U$-groups}

The structure of abelian linear groups over the integers of an algebraic number field has been described by Dade [2]. In this section we give a generalisation of his result to completely reducible $U$-groups.

2.1 Theorem. Let $A$ be an irreducible abelian $U$-group in $G L(n, F)$, and let the degree $[F: Q]$ of $F$ over $Q$ be $d$. Then $A$ is finitely generated, of rank at most $n d-1$, and $A_{T}$, the torsion subgroup of $A$, is cyclic of order $t$, where $\phi(t)$ (the Euler function) divides $n d$.

Note: the estimate for $\left|A_{T}\right|$ depends only on the fact that $A$ is an irreducible abelian subgroup of $G L(n, F)$, not on the condition $(U)$. 
Proof. Let $[A]$ be the linear hull of $A$ over $F$ (i.e. $[A]$ is the subalgebra of the full matrix algebra $M(n, F)$ generated by elements of $A)$. $[A]$ is irreducible, and therefore is a simple ring ([3], p. 56). Since $[A]$ is also a commutative ring with unity, the fact that it is simple makes it a field. Let $a_{1}, \cdots, a_{s}$ be a basis for $[A]$ over $F$. Let $V$ be an $n$-dimensional $F$-module, and identify $\operatorname{End}_{F} V$ and $M(n, F)$. Let $v \in V, v \neq 0$. Because $[A]$ is a field, $v a_{1}, \cdots, v a_{s}$, are linearly independent over $F$. They span a subspace $W$ of $V$ which is invariant under $[A]$. Since $[A]$ is irreducible, $W=V$, and the dimension $[[A]: F]$ of $[A]$ over $F$ is equal to $n$.

The group $A$ is therefore isomorphic to a subgroup of the multiplicative group of a finite extension $E$ of $F$ such that $[E: Q]=n d$. The condition $(U)$ satisfied by the elements of $A$ implies that each $a \in A$ corresponds to a unit in the integers of $E$. By Dirichlet's theorem on units in an algebraic number field ([4], Ch. XI), the rank of the group of units of $E$ cannot exceed $n d-1$.

Suppose $A$ has an element of order $r>1$. Then $E$ contains a primitive $r$-th root of unity, $\zeta$, say, and $\phi(r)=[Q(\zeta): Q]$ divides $[E: Q]=n d$. Since each finite multiplicative group in a field is cyclic, we conclude that $A_{T}$ is a cyclic group of order $t$ such that $\phi(t)$ divides $n d$. By an obvious argument we obtain the following corollary.

2.2 Corollary. A completely reducible abelian $U$-group in $G L(n, F)$ requires at most nd generators, where $d=[F: Q]$.

\section{Irreducible nilpotent $U$-groups}

Let $N$ be an irreducible nilpotent $U$-group in $G L(m, F)$.

3.1 THEOREM. If the class of $N$ is $c$, then

$$
c \leqq \begin{cases}2 m\left(1+\log _{2} d\right) & \text { if } d>1 \\ 2.12 m & \text { if } d=1\end{cases}
$$

where $d=[F: Q]$.

Note: This estimate depends only on the fact that $N \subseteq G L(m, F)$, not on the condition $(U)$.

Proof. Let $N=\gamma_{1}(N) \supset \gamma_{2}(N) \supset \cdots \supset \gamma_{c+1}(N)=1$ be the lower central series for $N$, and let $s$ be the smallest index such that $\gamma_{s}(N)$ is abelian. Since $\left[\gamma_{i}(N), \gamma_{j}(N)\right] \subset \gamma_{i+j}(N)$ we must have $s \leqq[c / 2]+1$.

By Clifford's theorem, $\gamma_{s}(N)$ is completely reducible over $F$. Suppose $\gamma_{s}(N)$ has $r$ homogeneous components. We shall show that $c \leqq 2 r\left(1+\log _{2} m d / r\right)$.

In any irreducible nilpotent linear group the index of the centre is finite ([1], p. 64). $|N: Z(N)|$ finite implies $\left|\gamma_{2}(N)\right|$ finite ([6], problem 5.24). If $s \neq 1$, $\gamma_{s}(N)$ is therefore finite, and is a subgroup of a direct product of $r$ cyclic groups of order $t_{1}$, where $\phi\left(t_{1}\right)$ divides $m d / r$. Let $Q_{s}$ be the Sylow $q$-subgroup of $\gamma_{s}(N)$, and suppose $\left|Q_{s}\right|=q^{l}$. Let $Q_{s+j}$ be the Sylow $q$-subgroup of $\gamma_{s+j}(N)$. Then 
$Q_{s+l}=1$. Let $p^{\alpha}$ be the highest prime power dividing $t_{1}$. Then for each $Q_{s}$, $l \leqq r \alpha$, and so $\gamma_{s+r \alpha}(N)=1$, and $c+1 \leqq s+r \alpha \leqq[c / 2]+1+r \alpha$. Hence $c \leqq 2 r \alpha$. Since $\phi\left(t_{1}\right)$ divides $m d / r, p^{x-1}$ divides $m d / r$, and so $\alpha-1 \leqq \log _{2} m d / r$. We have now $c \leqq 2 r\left(1+\log _{2} m d / r\right)$, and the result follows from this if we consider the maximum value of $2 x\left(1+\log _{2} m d / x\right)$ over $1 \leqq x \leqq m$.

3.2 Corollary. There exist maximal irreducible nilpotent $U$-groups in $G L(m, F)$.

This follows from 3.1 by an application of Zorn's Lemma.

3.3 Divisors of $|N: Z(N)|$ : All prime factors of $|N: Z(N)|$ divide the exponent $m_{2}$ of $Z_{2}(N) / Z(N)$, and $m_{2}$ divides $m$ ([1], Chapter III, Lemmas 19 and 22). Also, if $x Z(N)$ is of order $k$ in $Z_{2}(N) / Z(N)$, there exists $y \in N$ such that $[x, y]$ has order $k$ ([1], Chapter III, Lemma 20). In our case we have an additional condition on $m_{2}$. [x,y] lies in the torsion subgroup of $Z(N)$, which is cyclic, of order $t_{2}, m_{2}$ divides $t_{2}$, and $\phi\left(t_{2}\right)$ divides $m d$.

3.4 In particular, if $m d$ is odd, there are no non-abelian irreducible nilpotent $U$-groups in $G L(m, F)$.

Proof. If $m d$ is odd, $t_{2}=1$ or 2 , since $\phi\left(t_{2}\right)$ divides $m d$, and $m_{2}$ must be odd. Hence $m_{2}=1$ and $N$ is necessarily abelian.

3.5 Structure of $N / A$, where $A$ is a maximal normal abelian subgroup of $N$.

LEMMA. (i) N/A is isomorphic to a nilpotent permutation group $\hat{N}$ of degree $k$, where $k$ divides $m$.

(ii) If $N$ is primitive (see [5], p. 346), $\hat{N}$ is semiregular (i.e. a permutation group in which only the identity leaves any symbol fixed).

Proof. (i) $A$ is finitely generated. Choose a finite set of generators for $A$, and adjoin their eigenvalues to $F$. The field $E$ obtained is a normal extension of $F$. If we consider $N$ as a subgroup of $G L(m, E), N$ is completely reducible, and all its irreducible components are of equal degree. ([5], Theorems 69.4 and 70.15). $A$ is also completely reducible over $E$. Since $A$ is abelian, and each of a set of its generators can be diagonalised in $G L(m, E), A$ is reducible to a diagonal group.

Let $W$ be a minimal invariant space for $N$ in $V^{E}$ (where $V^{E}$ is an $m$-dimensional $E$-module, and we have identified $\operatorname{End}_{E} V$ with $\left.M(m, E)\right)$. Then $\operatorname{dim} W$ divides $m$. Let $\tau: x \rightarrow x \mid W$ (the restriction of $x \in N$ to $W$ ). Let $y \in \operatorname{ker} \tau \cap Z(N) . Z(N)$ is isomorphic to one of its own irreducible components. Hence $y \mid W=1$ implies $y=1$, and we have ker $\tau \cap Z(N)=1 . \tau$ is therefore faithful. Define $N^{*}=N \mid W$, $A^{*}=A \mid W$. We shall prove the result for $N^{*} / A^{*}$.

$A^{*}$ is reducible to a diagonal group. Let $W_{1}, \cdots, W_{k}$ be the distinct eigenspaces for $A$ in $W \cdot A^{*}\left|W_{1}, \cdots, A^{*}\right| W_{k}$ are the homogeneous components of $A^{*}$, and the spaces $W_{1}, \cdots, W_{k}$ are permuted by the elements of $N^{*}$ (see [5], p. 345). 
Since $A^{*}$ is its own centralizer in $N^{*}\left([6]\right.$, problem 6.36) we have $N^{*} / A^{*} \simeq \hat{N}$, a nilpotent permutation group on $k$ symbols. $k$ divides $\operatorname{dim} W$, which divides $m$.

(ii) If $N$ is a primitive group in $G L(m, F), A$ is isomorphic to one of its own irreducible components over $F$. Hence if $a \in A, a-1$ is either zero or invertible. If $x^{*} \in N^{*}$ fixes an eigenspace $W_{1}$ of $A^{*}$ in $W$, then $[x, a] \mid W_{1}=1$ for all $a \in A^{*}$. This implies $[x, a]=1$ for all $a \in A$, and so $x \in A . \widehat{N}$ is therefore semiregular.

3.6 In particular, suppose $\hat{N}$ is transitive, and $E=Q$ or $Q(\theta)$, where $\theta$ is complex of degree 2 over $Q$. Then $N$ is finite.

Proof. $Z\left(N^{*}\right)$ is a group of scalar matrices $f \cdot 1, f \in E$, if $\hat{N}$ is transitive. By Dirichlet's Theorem, the group of units of $E$ is finite, and so $Z\left(N^{*}\right)$ is finite. Hence $Z(N)$ and $|N: Z(N)|$ are both finite, and the result follows.

3.7 THEOREM. If the class of $N$ is 2, then $N$ has a faithful absolutely irreducible representation in $G L(s, E)$ where $E$ is the field defined in 3.5 , and $s$ divides $m$. It follows that $|N: Z(N)|=s^{2}$.

Proof. We shall show that the group $N^{*}$ defined in 3.6 is absolutely irreducible.

(i) If the class of $N$ is $2, \hat{N}$ is semiregular. For: Let $W$ be the space defined in 3.5, and $W_{1}$ an eigenspace for $A^{*}$ in $W$. We have already: if $x^{*}=x \mid W \in N^{*}$ fixes $W_{1}$, then $[x, a] \mid W_{1}=1$ for all $a \in A$. Since the class of $N$ is $2,[x, a] \in Z(N)$. $Z(N)$ is isomorphic to one of its own irreducible components, and so $[x, a]=1$ for all $a \in A$. Hence $x \in A$, and $\hat{N}$ must then be semiregular.

(ii) Let $w \neq 0 \in W_{1}$ and let $1=x_{1}, x_{2}, \cdots, x_{5}$ be a complete set of coset representatives for $A$ in $N$. Let $L_{1}$ be the space spanned by $w, x_{i}^{*}=x_{i} \mid W$, and define $L_{j}=L_{1} x_{j}^{*} j=1, \cdots, s$. By (i) the $L_{j}$ belong to distinct eigenspaces of $A^{*}$ in $W$. They are permuted transitively by the elements of $N^{*}$. The space $L=L_{1} \oplus \cdots \oplus L_{s}$ is a nonzero invariant space for $N^{*}$ in $W$, and so $L=W$.

The construction of $L$ shows that the centralizer of $N^{*}$ in $M(s, E)$ can contain scalar matrices only. $N^{*}$ is therefore the required representation of $N$. (see [5], p. 202).

(iii) $|N: Z(N)|=s^{2}$. This can be deduced from [1] Chapter I, Lemma 10 . The following more elegant argument is due to Professor J. D. Dixon.

The linear hull of $N^{*}$ over $E$ has dimension $s^{2}$ ([5], Theorem 27.8). We can therefore find elements $x_{1}^{*}, \cdots, x_{s^{2}}^{*} \in N^{*}$ that form a basis for $M(s, E)$. Since $Z\left(N^{*}\right)$ is a group of scalar matrices, $x_{1}^{*}, \cdots, x_{s^{2}}^{*}$ are in distinct cosets of $Z\left(N^{*}\right)$ in $N^{*}$. We show that they form a complete set of coset representatives for $Z\left(N^{*}\right)$ in $N^{*}$.

Let $x^{*} \in N^{*}, x^{*} \notin Z\left(N^{*}\right)$. Then there exists $y^{*} \in N^{*}$ such that $\left[x^{*}, y^{*}\right]=$ $z^{*} \in Z\left(N^{*}\right), z^{*}=\zeta \cdot 1, \zeta \neq 1$, i.e. $\left(y^{*}\right)^{-1} x^{*} y^{*}=\zeta x^{*}, \zeta \neq 1$. Trace $x^{*}=$ trace $\left(y^{*}\right)^{-1} x^{*} y^{*}=\operatorname{trace} \zeta x^{*}=\zeta$ trace $x^{*}$. Since $\zeta \neq 1$, trace $x^{*}=0$. Now let $x^{*}$ be 
any element of $N^{*} . x^{*}=\sum_{i=1}^{s^{2}} \alpha_{i} x_{i}^{*}, \alpha_{i} \in E$. At least one $\alpha_{j} \neq 0$. Trace $x^{*}\left(x_{j}^{*}\right)^{-1}$ $=\sum_{i=1}^{s^{2}} \alpha_{i}$ trace $\left(x_{i}^{*}\left(x_{j}^{*}\right)^{-1}\right)=s \alpha_{j} \neq 0$. As we have just seen, this implies $x^{*}\left(x_{j}^{*}\right)^{-1} \in Z\left(N^{*}\right)$, and gives the result.

\section{Completely reducible soluble $U$-groups}

4.1 Let $S$ be an irreducible soluble $U$-group in $G L(n, F)$. Suppose $S$ is maximal with respect to the property of being soluble.

Suppose $S$ is imprimitive. Let $V$ be an $n$-dimensional $F$-module, and identify End $_{F} V$ with $M(n, F)$. Let $V=V_{1} \oplus \cdots \oplus V_{k}$ be a complete decomposition of $V$ into systems of imprimitivity for $S$ (cf. [1], p. 7). By an argument similar to that used in the proof of Lemmas 3 and 4 of [1], Chapter I, $S$ has a normal subgroup $G$ for which each $V_{i}, i=1, \cdots, k$, is an invariant space, such that $S / G$ is isomorphic to a maximal soluble permutation group of degree $k . G$ is the direct product of the groups $G \mid V_{i}, i=1, \cdots, k$. Each $G \mid V_{i}$ is isomorphic to $G \mid V_{1}$, which is a maximal irreducible primitive soluble $U$-group in $G L(n / k, F)$.

4.2 Let $S$ be a primitive irreducible soluble $U$-group in $G L(n, F)$. We describe $S$ by describing the factors in the series

$$
1 \triangleleft A \triangleleft B \triangleleft C \triangleleft S
$$

where $A$ is a maximal normal abelian subgroup of $S, C$ the centraliser of $A$ in $S$, and $B$ the Fitting subgroup of $C$. Suprunenko ([1], Chapter I) uses a similar decomposition to describe primitive soluble linear groups, except for a different choice of $B$. Our choice of $B$ allows us to use information about irreducible nilpotent $U$-groups.

4.3 The group $A$ : By Clifford's Theorem $A$ is completely reducible over $F$. Since $S$ is primitive, all the irreducible components of $A$ are equivalent, and so $A$ is isomorphic to an irreducible abelian $U$-group in $G L(t, F)$, where $t$ divides $n$. The results of 2.1 then apply to $A$.

4.4 LemMA. $B$ is nilpotent, of class at most 2.

Proof. The Fitting subgroup of any linear group is nilpotent ([9], Theorem 1 (ii)). Let the class of $B$ be $c, B=\gamma_{1}(B) \supset \gamma_{2}(B) \supset \cdots \supset \gamma_{c+1}(B)=1$ the lower central series for $B$, and $r$ the smallest index such that $\gamma_{r}(B)$ is abelian. $\gamma_{r}(B) \subset C$, and so $\gamma_{r}(B) \cdot A$ is abelian, and normal in $S$. By the maximality of $A$, $\gamma_{r}(B) \subset A=Z(B)$. We have therefore $c \leqq r$, and, by the argument used in 3.1, $r \leqq[c / 2]+1$. Hence $c \leqq 2$.

4.5 Since $S$ is primitive, $B$ is isomorphic to one of its own irreducible components, and so, if $c=2$, we can apply 3.7 , with $s$ a divisor of $n / t$ (where $t$ is the degree of an irreducible component of $A$ ). The primes dividing $s$ must satisfy the conditions of 3.3 . 
4.6 In particular, if nd is odd, $B=A=C=H$, where $H$ is the Fitting subgroup of $S$.

Proof. By 3.4, nd odd implies $B$ and $H$ are both abelian. If $C \neq A, C / A$ contains a non-trivial characteristic abelian subgroup $K / A$, and $K$ is necessarily nilpotent, giving a contradiction.

4.7 The group $B / A$ : Suppose $B / A$ is non-trivial. Since $B$ is the Fitting subgroup of $C, B / A$ is the maximal normal abelian subgroup of $C / A . B / A$ is equal to its own centralizer in $C / A$ (cf. [1], Chapter I, proof of Theorem 4). By [1], Chapter I, Lemma 15, the Sylow $q$-subgroups of $B / A$ are elementary abelian $q$-groups.

4.8 The groups $C / B$ and $S / B$ : By [1], Chapter I, Theorem 11, if $s=q_{1}^{\alpha_{1}} \cdots q_{k}^{a_{k}}$, $C / B$ is isomorphic to a soluble subgroup of the direct product of the symplectic groups $\mathrm{Sp}\left(2 \alpha_{1}, q_{1}\right), \cdots, \operatorname{Sp}\left(2 \alpha_{k}, q_{k}\right)$.

By an argument similar to that of 3.5 we obtain: $S / C$ is isomorphic to a soluble semiregular permutation group of degree $t$. (cf. [1], p. 12). For these two factors we obtain no special restrictions.

4.9 THEOREM. A completely reducible soluble U-group $S$ in $G L(n, F)$ satisfies the maximum condition for subgroups.

We shall prove the equivalent condition that all subgroups of $S$ are finitely generated.

Proof. (i) If $S$ is a primitive irreducible soluble $U$-group, it is a finite extension of a finitely generated abelian group, and the result follows. This extends to the maximal imprimitive irreducible case by 4.1 , and therefore to any irreducible soluble $U$-group in $G L(n, F)$.

(ii) If $S$ is completely reducible, with $V=V_{1} \oplus \cdots \oplus V_{k}$ a direct sum of minimal $S$-invariant subspaces, then $S$ is isomorphic to a subgroup of $S \mid V_{1} \times$ $S\left|V_{2} \times \cdots \times S\right| V_{k}$. Each $S \mid V_{i}, i=1, \cdots, k$, is an irreducible soluble $U$-group in $G L\left(n_{i}, F\right)$, where $n_{i}=\operatorname{dim} V_{i}$. The result then holds for each $S \mid V_{i}$, and hence for $S\left|V_{1} \times \cdots \times S\right| V_{k}$ and for $S$.

4.10 COROLLARY. If $S$ is any completely reducible soluble $U$-group in $G L(n, F)$, we can apply two theorems of Hirsch to conclude:

(i) $S$ is polycyclic [7], p. 193.

(ii) If $S$ is infinite, $S$ has a normal subgroup $H$ such that $|S: H|$ is finite, and $H$ has a normal series $H=H_{0} \supset H_{1} \supset \cdots \supset H_{k}=1$, in which each factor $H_{i-1} / H_{i}, i=1, \cdots, k$, is an infinite cyclic group [8], p. 188. We can actually take $H$ to be a finitely generated torsion-free abelian group, since we have a bound on the orders of torsion elements in a maximal normal abelian subgroup of finite index in $S$. 
Note. It follows from Mal'cev's Theorem ([1], p. 31) that any completely reducible soluble linear group is an extension of an abelian group by a finite group. 4.9 and 4.10 can therefore be made to follow directly from 2.1 (but without intermediate results 4.4-4.6). I am indebted to the referee for this comment.

\section{References}

[1] D. A. Suprunenko, Soluble and Nilpotent Linear Groups (Translations of Math. Monographs, Vol. 9, Amer. Math. Soc., Rhode Island, 1963).

[2] E. C. Dade, 'Abelian Groups of Unimodular Matrices', Illinois J. Math. 3 (1959), 11 - 27.

[3] M. Burrow, Representation Theory of Finite Groups (Academic Press, 1965).

[4] H. Pollard, Algebraic Number Theory (Wiley 1961).

[5] C. W. Curtis and I. Reiner, Representation Theory of Finite Groups and Associative Algebras (Interscience, 1962).

[6] J. D. Dixon, Problems in Group Theory (Blaisdell, 1967).

[7] A. Kurosh, Theory of Groups (Chelsea, 1958).

[8] K. A. Hirsch, 'On Infinite Soluble Groups (III)', Proc. Lond. Math. Soc. (2) 49 (1946), 184194.

[9] B. A. F. Wehrfritz, 'Locally Nilpotent Linear Groups', J. London Math. Soc. 43 (1968), 667-674.

University of New South Wales 